\title{
BEAM-BASED OFFSET CALIBRATION OF THE PLS BPM
}

\author{
J. Y. Huang, D. H. Jung, K. H. Kim, S. H. Nam, M. Yoon and I. S. Ko \\ Pohang Accelerator Laboratory, POSTECH \\ Pohang 790-784, Korea
}

\begin{abstract}
There are 108 beam position monitors in the PLS for the orbit measurement and closed orbit correction. As the BPM pickup electrodes are assembled directly on the $10 \mathrm{~m}$-long vacuum chamber, calibration of the electrical center of BPMs have been done with the external rf transmission-reflection method. Since the closed orbit distortion is still larger than $500 \mu \mathrm{m} \mathrm{rms,} \mathrm{we} \mathrm{are}$ developing the beam-based calibration method for the BPMs in PLS to improve the accuracy of the absolute position reading. In this report we present the method of beam-based calibration of BPMs and its experimental results.
\end{abstract}

\section{INTRODUCTION}

The Pohang Light Source (PLS) is a dedicated third generation synchrotron light source with nominal beam energy of $2 \mathrm{GeV}$. In the low emittance storage ring like PLS, minimization of the closed orbit distortion is a necessary condition for the optimum performance of the machine. To minimize the closed orbit distortion, the alignment accuracy of the accelerator component has to be within $150 \mu \mathrm{m}$. Novel opto-mechanical methods have been used for the survey and alignment of the lattice magnets and BPMs in the PLS. Local alignment accuracy is less than $100 \mu \mathrm{m}$ rms [1]. Among the accelerator components, BPMs and magnets are particular ones which have magnetic or electrical center different from the mechanical center. In view of the charged particles circulating in the storage ring, the true centers of the lattice components are magnetic or electrical centers, not the mechanical ones. The magnetic center of each magnet was precisely measured within $20 \mu \mathrm{m}$ on the magnetic field measurement table before the installation. However, BPMs could not be calibrated individually in the PLS, because the BPMs are plugged into the $10 \mathrm{~m}$ long single piece vacuum chamber. Instead, we have measured all the coupling coefficients between the pickup electrodes using a network analyzer and a $48 \mathrm{~dB}$ gain power amplifier to find the relative gains of the pickup electrodes and the electrical centers of the BPM.

However, since the coupling coefficients are very sensitive to the chamber structure, it seems that this method is not as accurate as done on the test bench [2]. Indeed, the closed orbit distortion after the closed orbit correction is no better than $500 \mu \mathrm{m}$ in the PLS. Therefore, we believe the rms error of the electrical offset measurement of the BPMs should be larger than $500 \mu \mathrm{m}$.

Recently, beam-based alignment (BBA) techniques by using quadrupole magnets are tried in several laboratories [3,4,5]. In the PLS, we are also developing the beam-based alignment technique for the calibration of electrical offsets of selected BPMs. In particular, we try to do a beam-based alignment which is rather geometrical method derived from simple beam dynamics. For the preliminary test, we installed 14 quadrupole magnet current shunt circuits in one cell of the storage ring [6]. In this paper, we describe on the preliminary tests and results of the BBA experiments.

\section{BEAM BASED ALIGNMENT}

The purpose of the BBA is the measurement of the offset of electrical center of the BPM from an ideal reference orbit or the magnetic center of the nearest quadrupole magnet. If the BPM is located at the center of the quadrupole magnet, or even when the BPM is located very close to the quadrupole magnet as shown in Fig. 1, the deviation $d \theta_{Q}$ between the reference orbit and the beam trajectory is very small or even eliminated by taking average over the many beam trajectories passing through the center of the quadrupole magnet. In this case, the offset $\Delta x_{P M}$ is just the average of the beam position $\left\langle x_{P M}\right\rangle$ read by the BPM when the orbit passes through the center of the quadrupole magnet.

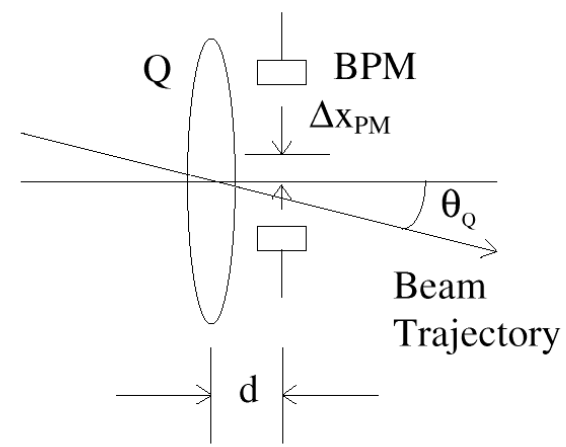

Figure 1: When the distance $d$ between quadrupole magnet and BPM is small compared to the betatron wavelength or the crossing angle $\theta_{Q}$ is negligible by taking average over many beam trajectories $\left(\left\langle d G_{Q}\right\rangle \sim 0\right)$, the BPM offset $\Delta x_{P M}$ is equivalent to the average of beam position $\left\langle x_{P M}\right\rangle$.

For the test, we consider that the initial beam position is $x_{Q}$ at the quadrupole magnet, and $x_{P M}$ at the BPM. A corrector magnet and another BPM2 are also selected. These are well apart from the quadrupole magnet. By changing the corrector magnet current, the beam position $x_{Q}$ at the quadrupole magnet can be controlled. When the quadrupole magnet strength is changed by $\delta k_{Q}$, the beam experiences the change of kick angle $\delta \sigma_{Q}$ such as

$$
\delta 6_{Q}=\delta k_{Q} x_{Q} l_{Q}
$$


Consequently, the closed orbit change $\delta x_{P M 2}$ at BPM2 is

$$
\delta x_{P M 2}=\delta 6_{Q}\left(\beta_{Q} \beta_{P M 2}\right)^{1 / 2} \cos \left(\varphi_{Q}-\varphi_{P M 2}+\pi v\right) / 2 \sin (\pi v),
$$

where $l_{\mathrm{Q}}$ is the effective length of the quadrupole magnet and $\beta_{Q}$ is the betatron function at the quadrupole magnet. If the orbit passes through the center of the quadrupole magnet, $x_{O}=0$ and, accordingly, $\delta x_{P_{M 2}}=0$. The offset $\Delta x_{P M}$ of the BPM is then equivalent to the average of the beam position reading $\left\langle x_{P M}\right\rangle$.

In some accelerators like the PLS, however, BPMs are not located close to the quadrupole magnets. The average $\left\langle d \sigma_{Q}\right\rangle$ is not negligible if the distance from the quadrupole magnet $d$ is large. Then we need a reference orbit or a reference trajectory, instead of a closed orbit, to measure the absolute BPM offsets. The closed orbit is not uniquely defined in a storage ring, because it changes with the change of lattice parameters, such as tunes. We will here define a unique reference trajectory by connecting the centers of the quadrupole magnets piecewise as shown in Fig. 2. The offset of the BPM is then defined as the offset from the reference trajectory. In the practical sense, the reference trajectory defined as above may be almost the same to the design orbit or the closed orbit, because the overall alignment accuracy of the storage ring components are less than $150 \mu \mathrm{m}$ rms.

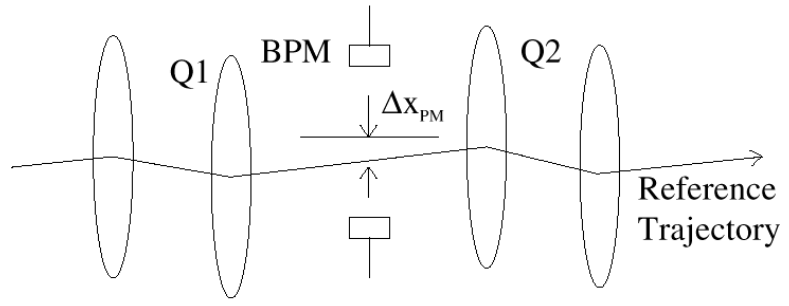

Figure 2: A reference trajectory is defined as the piecewise straight lines connecting the centers of the quadrupole magnets.

Measurement of the absolute offset of BPMs can be realized using two quadrupole magnets. First, we find the orbit which passes through the center of the quadrupole magnet Q1 near the BPM. Beam position $x_{P M}$ measured by BPM is not the offset of the BPM in this case, because the beam will cross the quadrupole magnet center with an angle $\theta_{Q I}$

$$
\epsilon_{Q 1}=x_{P M} / d_{l}=x_{Q 2} / d
$$

where $x_{Q_{2}}$ is the beam position at Q2 and $d$ is the distance between two quadrupole magnets $\mathrm{Q} 1$ and Q2. To measure $\theta_{Q 1}, x_{Q^{2}}$ should be measured first. It can be done by measuring the change of beam position $\delta x_{P M}$ at the BPM with respect to the change of the focusing strength $\delta$ $k_{Q^{2}}$ of Q2. Then $x_{Q^{2}}$ can be obtained from Eq. (2) as

$$
\begin{aligned}
x_{Q 2}= & 2 \delta x_{P M} \sin (\pi v) /\left[\delta k_{Q 2} l_{Q 2}\left(\beta_{Q 2} \beta_{P M}\right)^{1 / 2}\right. \\
& \left.\times \cos \left(\varphi_{P M}-\varphi_{Q 2}+\pi v\right)\right] .
\end{aligned}
$$

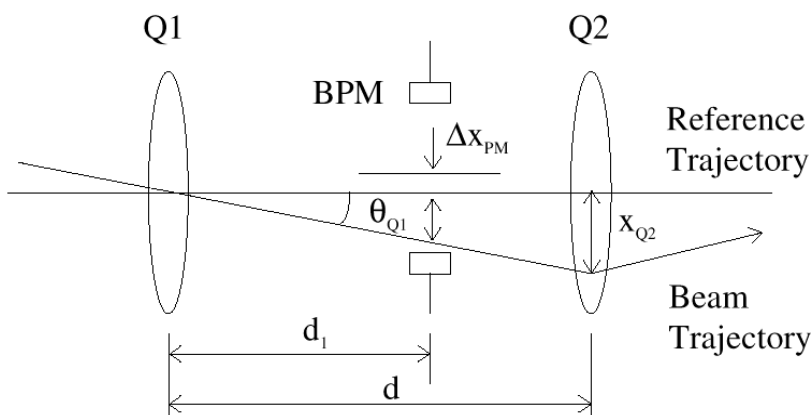

Figure 3: Difference of the beam trajectory and the reference trajectory, $d_{l} \theta_{Q l}$, can be measured with two quadrupole magnets Q1 and Q2.

In Fig. 3, the offset $\Delta x_{P M}$ of the BPM from the reference trajectory is finally

$$
\begin{aligned}
\Delta x_{P M}= & x_{P M}-d_{1} \theta_{Q 1} \\
= & x_{P M}-d_{1} x_{Q 2} / d \\
= & 2 d_{1} \delta x_{P_{M}} \sin (\pi v) /\left[\delta k_{Q_{2}} d l_{Q^{2}}\left(\beta_{Q_{2}} \beta_{P M}\right)^{1 / 2}\right. \\
& \left.\times \cos \left(\varphi_{Q^{-}}-\varphi_{P M}+\pi v\right)\right] .
\end{aligned}
$$

We again take the average over the many beam trajectories passing through the center of the quadrupole magnet using the selected corrector magnets. In these analysis, the tune shift by the change of quadrupole magnet strength is neglected.

\section{EXPERIMENTS}

We have done an experiment as a preliminary test for the application of BBA to the PLS storage ring. Two focusing quadrupole magnets, P05Q1D and P06Q1U, at both sides of the BPM 6PM2, and a corrector magnet P05CH6 are used for the experiment. A bending magnet located in between two quadrupole magnets is regarded as the free drift space. Two corrector magnets, $\mathrm{P} 06 \mathrm{CH} 1$ and $\mathrm{P} 06 \mathrm{CH} 2$, located in between two quadrupole magnets are turned off. Fig. 3 is, therefore, equivalent to our experimental setup.

Current shunt circuits for 14 quadrupole magnets of one lattice period of the storage ring are installed for the experiment. It is controlled linearly within $10 \%$ of the quadrupole magnet strength. Since the data acquisition and control system is not completed yet, each shunt circuit is manually controlled for this experiment.

The closed orbit is changed by the corrector magnet P05CH6 to change the beam position at the quadrupole magnet Q1 (P05Q1D). Changes of the beam position $\delta$ $x_{P M}$, between the shunt current on and off, are measured and plotted with respect to the beam position $x_{P M}$. By fitting the data, the closed orbit passing through the center of Q1 is found. In Fig. 4, $x_{P M}=2.5843 \mathrm{~mm}$ with the fitting accuracy better than $170 \mu \mathrm{m}$ rms. To find beam position $x_{Q^{2}}$ at $\mathrm{Q} 2$ when the beam passes through the center of Q1, the change of beam position $\delta x_{P M}$ at the BPM is measured by changing the focusing strength $\delta k_{Q^{2}}$ of Q2. In Fig. 5, we get $\delta x_{P M} / \delta k_{Q 2}=-1.775$, and $x_{Q 2}$ is calculated from Eq. (3) as $1.637 \mathrm{~mm}$. The linearity of the quadrupole magnet current shunt circuit, tested with the beam, is sufficiently 
good as shown in Fig. 5. Finally, the true offset of the BPM can be calculated from Eq. (4). With $d_{l}=6.813 \mathrm{~m}$ and $d=7.04 \mathrm{~m}$, we obtain $\Delta x_{P M}=0.947 \mathrm{~mm}$.

\section{CONCLUSION}

We have done an experiment as a preliminary test for the application of BBA to the PLS storage ring. Two defocusing quadrupole magnets P05Q1D, P06Q1U and a BPM 6PM2 are used for the experiment.

Results of this experiments are shown in Figs. 4 and 5 , where $\Delta x_{P M}=0.947 \mathrm{~mm}$ with the fitting accuracy $170 \mu$ $\mathrm{m}$ rms. The linearity of the shunt circuit is sufficiently good for the purpose of beam-based alignment. As we expected before the test, the electrical offset of the BPM seems to be large.

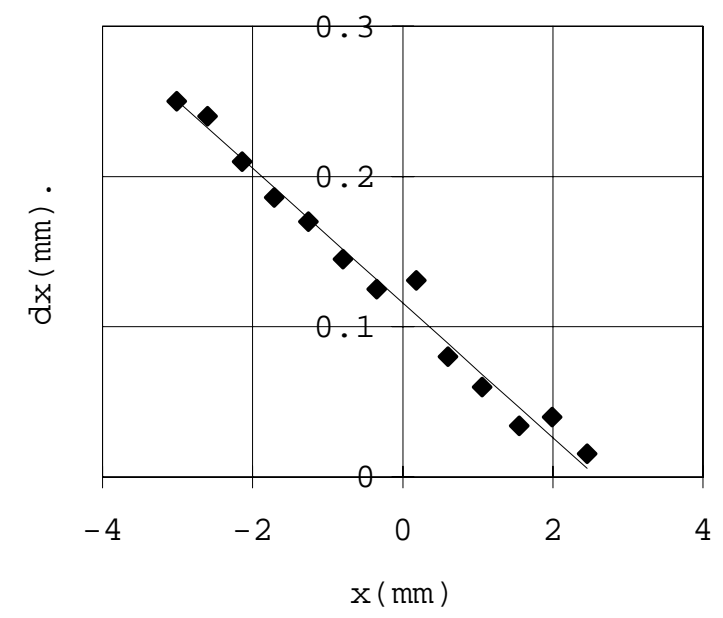

Figure 4: Plot of beam positions at the BPM and change of beam position by turn on and off the quadrupole magnet shunt current.

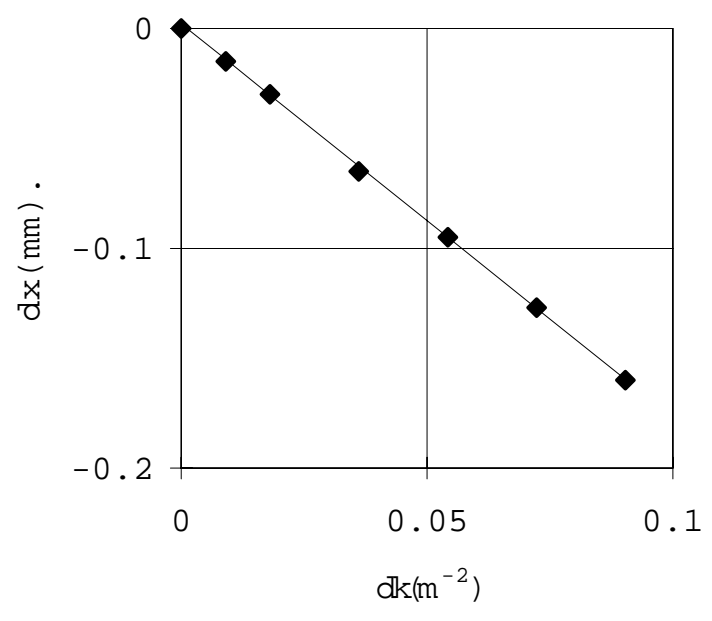

Figure 5: Change of beam position vs change of quadrupole magnet strength. Nominal focusing strength of the quadrupole magnet $k_{0}$ is $-0.64178 \mathrm{~m}^{-2}$

Since the shunt circuits were controlled manually, sufficient averaging of the data was not taken. To verify the consistency of the BBA technique in the PLS, we have to do the same experiment in the reverse direction. The same offset values should be obtained for the same BPM.

All the new current shunt circuits are completed and are being installed now [6]. The shunt current is linearly variable up to $10 \%$ of the quadrupole magnet current. By the end of this year, all the current shunt circuits will be controlled with RS232 from the local VME-bus MIU (machine interface unit). Each MIUs will be controlled offline from the main PLS control system via the independent Ethernet which has been installed for the development of the low level MIU program. Control software for the system is now under developing. Several precision BPMs are also prepared for the test. When all these are installed, more precise BBA will be possible.

\section{REFERENCES}

[1] K. W. Seo et. al., "Precision Surveying and Smoothing Analysis for Pohang Light Source," in this proceedings.

[2] J. M. Hinkson, ALS, private communications.

[3] Barnett et. al., "Dynamic Beam Based Alignment," $2^{\text {nd }}$ European Workshop on Beam Diagnostics and Instrumentation for Particle Accelerators, DESY M-95-07 (1995)

[4] K. Endo et. al., "Preliminary Orbit Measurement for Beam-Based Alignment," Proc. of 1996 EPAC, Sitges (1996).

[5] P. Tenenbaum et. al., "Beam-Based Magnetic Alignment of the Final Focus Test Beam," Proc. of 1995 PAC, Dallas (1996).

[6] S. H. Nam et. al., "A High Current Shunt Regulator for Quadrupole Magnets in PLS 2GeV Storage Ring," in this proceedings. 